\title{
An Analysis of Gifted Teachers' Interest in the Standards of National Gifted Education Program
}

\author{
Woo Suk Jin ${ }^{1}$ \\ ${ }^{1}$ Ph.D., Cooperative Course for Gifted Education, Busan National University, Graduate School, \\ jwsjws@empal.com
}

\begin{abstract}
The purpose of this study is to analyze the differences in interest by background variables using CBAM models as a study on the analysis of gifted teachers' interest in national gifted program criteria. For this purpose, a survey was conducted on 200 teachers participating in gifted education in the $\mathrm{K}$ area and the results were utilized. The conclusions of this study are as follows. First, the overall interest in the national gifted program was high, and gifted teachers were positive about the overall impact and outcome of the national gifted program on students in gifted educational activities. Therefore, it will be necessary to utilize and support specific teacher training and gifted education so that the standards of the national gifted program can be applied to the educational site properly in the educational field. Second, national gifted curriculum programs customized for each student level should be developed and distributed based on understanding of the national gifted program standards. Training support and provision of materials should be prioritized, along with suggesting specific writing methods, so that teachers in charge of gifted education can write systematic programs rather than piecemeal education programs. Third, the effective and successful use and settlement of the national gifted education program standards will require continuous support for the dissemination of data and training of gifted education programs to gifted teachers through the development of regular gifted teaching.
\end{abstract}

Keywords: National Gifted Education Program Standards, CBAM, Gifted Education, Gifted Education Teachers, Interests

\section{Introduction}

Despite the quantitative expansion of gifted education, questions about the need to strengthen the quality aspects such as how to operate each institution, differences in programs, and teacher capabilities have been continuously raised. In the process of establishing the 3rd General Plan for Gifted Education, a plan was proposed to prepare detailed evaluation criteria such as step-by-step teaching methods, education instructors, and textbook utilization to verify the educational effectiveness of each component. This is to provide gifted students with differentiated education programs beyond those offered in regular schools for self-realization and social development. The regular curriculum focuses on knowledge, function, and attitude, but gifted education focuses on the development of creative problem solving, self-directed learning attitude, and leadership. However, the programs currently used in the field of gifted education tend to focus on certain areas depending on the major areas of teachers in charge of gifted education, and there are many overlapping and omitted contents.

As a result, standards were established to provide common guidelines at the national level for developing and operating gifted education programs, considering the need for gifted education policies and characteristics and conditions at the gifted education site. Since the national gifted program

Received: November 26, 2020; $1^{\text {st }}$ Review Result: January 15, 2021; $2^{\text {nd }}$ Review Result: March 02, 2021 Accepted: March 29, 2021 
standards can be successfully used in the field and lead to innovation in gifted education, continuous research on the national gifted program standards can be conducted through on-site training, and case studies are emphasized. Therefore, this study will be used as a basic research material to analyze the interest of teachers in charge of gifted education in terms of national gifted program standards and to investigate ways to raise interest and practice according to the results.

\section{Theoretical Background}

\subsection{Definition of Giftedness}

The definitions and concepts of giftedness are used differently by gifted scholars or by specific times, and from various perspectives. The definition of giftedness is used differently without obtaining a logically agreed uniform definition. As such, the definitions of giftedness use different approaches, so it is necessary to examine them accurately. Generally classified definitions of giftedness can be divided into six categories: psychometric definitions, characteristic-based definitions, social needs-based definitions, education-based definitions, special talents definitions, and multi-dimensional definitions[1].

\subsection{National Gifted Education Program: Science in Elementary and Middle School}

Programs as a teaching plan used in science gifted education should be able to provide opportunities for gifted students to express and utilize their various abilities, such as familiarity with abstraction, excellent concentration and persistence, curiosity about new things, quick understanding, and transition[2]. "National Gifted Education Program Standards: Elementary and Middle School Science" provided science gifted students with basic qualifications and abilities as scientists by presenting scientific concepts and practical exploration, as well as nature, integration concepts, convergence capabilities, and career factors[3].

\subsection{National Gifted Education Program: Elementary and Middle School Mathematics}

The National Gifted Education Program : The Elementary and Middle School Mathematics Composition System is a guide to the development and composition of programs aimed at fostering creative mathematic talent based on regular curriculum. To this end, the relevant major system was selected to correspond to the core concepts of mathematics and curriculum, and these contents were divided into I-level (basic), II-level (development), and III-level (expansion) and presented step-by-step. Using this, each gifted educational institution developing a gifted mathematics education program could decide the ratio of education activities to be included in the subject and subject-specific gifted programs, considering the level of students in the institution.

\subsection{CBAM Model}

The CBAM can investigate changes and innovations in education, and close diagnostics can be used for stages of interest, level of use, and Innovation configuration, and the results of which can be used to systematically improve the curriculum[4].

CBAM is recognized as a progressive interest-based process and is a model that embraces new changes and innovations, and describes the characteristics and patterns of interest that are identified in implementing changes in curriculum innovation and school improvements. The system considers the role of people in the initiation and implementation of new changes and innovations, and presupposes 
that educational sites can be improved when people accept, implement, and change. In particular, the improvement and change of schools depends on the interest and understanding of teachers at school sites, so it is difficult to make changes in schools without proper understanding and interest in them[5].

The level of interest can be divided into seven levels, each, information, personal, operational, consequential, cooperative, and reinforcement, and as individuals encounter new innovations and changes, they think that the seven levels of interest strongly emerge[6].

In addition, individual interests develop in the process of change and innovation, which they have developed from zero to six levels. Factors to consider in determining innovation and change in the curriculum can be described as administrative and financial support for education as a whole, on-site education programs, teachers, learners, and school curricula, but above all, the quality of education in school field classrooms depends on teachers' abilities and interests[7].

These CBAMs have been identified and utilized for a long time through research on school curriculum innovation and change[8].

With these characteristics, CBAM is appropriate to describe the process of introducing and implementing the latest education methods or technologies at new educational sites, and most of the research on the curriculum in Korea[9].

CBAM understands the acceptors in the process of change and innovation and can be used to provide appropriate support through these courses including the stages of interest (SoC), innovation configuration map (IC map), and levels of execution (LoU). At the implementation level, it is a tool that helps to manipulate the levels at which new changes and innovations are implemented, and a changetype map is to present steps 0 to 6 of interest in change and innovation in the form of profiles[10].

These three types of diagnostic tools can be described as interest-based acceptance models.

\section{Research Methods}

\subsection{Subject to Study}

In this study, 223 male and female gifted education teachers who participated in gifted education activities in K-area in 2019 were selected for the study to find out the interest of gifted teachers in the national gifted program. The general background of gifted teachers who responded to the survey is shown in [Table 1].

[Table 1] Distribution of Research

\begin{tabular}{|ccccc|}
\hline Separate & Separate & Headcount & $\%$ \\
\hline \hline \multirow{3}{*}{ By gender } & Man. & & 142. & 71. \\
& A woman. & & 58. & 29. \\
& & Total. & 200. & 100. \\
\hline \multirow{2}{*}{ School class } & Elementary School Teacher. & 150. & 75. \\
& Middle school teachers. & & 50. & 25. \\
\hline By establishment & & Total. & 200. & 100. \\
& State, public. & & 170. & 85. \\
\hline All & Private. & & 30. & 15. \\
\hline Support & & Total. & 200. & 100. \\
\hline
\end{tabular}




\begin{tabular}{|c|c|c|c|c|}
\hline & More than 16 classes. & & 66. & 33. \\
\hline & & Total. & 200. & 100. \\
\hline \multirow{6}{*}{$\begin{array}{c}\text { Production } \\
\text { A career }\end{array}$} & 0 - four years. & & 70. & 35. \\
\hline & 5 - nine years. & & 40. & 20. \\
\hline & 10 - 14 years. & & 20. & 10. \\
\hline & 15 - 19 years. & & 50. & 25. \\
\hline & For more than 20 years. & & 20. & 10. \\
\hline & & Total. & 200. & 100. \\
\hline \multirow{3}{*}{ Final education } & Academy. & & 114. & 57. \\
\hline & More than a master. & & 86. & 43. \\
\hline & & Total. & 200. & 100. \\
\hline \multirow{3}{*}{ The job } & Principles. & & 150. & 75. \\
\hline & A vocational teacher. & & 50. & 25. \\
\hline & & Total. & 200. & 100. \\
\hline \multirow{3}{*}{ The field } & Science. & & 152. & 76. \\
\hline & Math. & & 48. & 24. \\
\hline & & Total. & 200. & 100. \\
\hline
\end{tabular}

\subsection{Research Tools}

\subsubsection{Inspection Tools}

The questionnaire used in this study consisted of a background variable for a typical teacher identifying interest in national gifted program standards. The questionnaire measuring gifted teachers' interest was translated into the Stage of Concern Questionnaire (SoCQ) developed by Hall \& Hord (2006) and adapted and supplemented to the context of the national gifted program criteria to serve the purpose of the study. The Interest Measurement Questionnaire consists of 35 questions that can indicate the degree of interest in the national gifted program standards. The questionnaire consists of seven steps from level 0 to level 6, and each step consists of questions as shown in [Table 2].

[Table 2] Each step-by-step Statement

\begin{tabular}{|c|c|c|}
\hline \multicolumn{2}{|c|}{ Phase of interest } & Number of the sentence \\
\hline Perceptual interest & One step (perception) & $312,2223,330$. \\
\hline The self & Intelligence (information interest) & $6,14,15,235$. \\
\hline The self & Two steps (personal interest) & $713,17,233$. \\
\hline Challenges & Three steps (operational interest) & $4,816,234$. \\
\hline Impact(impact) & These are the results of the results & $11119,232$. \\
\hline Impact(impact) & Interactive interest & $5,10,18,229$. \\
\hline Impact(impact) & Sixty-six steps & $2,920,231$. \\
\hline
\end{tabular}

This researcher used quantitative research methods and secured the validity of the questionnaire through a review by two curriculum professors, three scholars, and five gifted education experts.

\subsubsection{Data Processing Method}

Data processing was analyzed using the IBM SPSS 23 program. First, to analyze the overall interest 
of teachers in charge of gifted education in terms of the national gifted education program standards, a descriptive statistical analysis of relative strength by stages was conducted on gifted teachers. Second, we analyzed whether there are different levels of interest for each individual depending on the general background of teachers in charge of gifted students (by gender, school grade, founder, overall academic supply, teaching career, final education, and position). The questionnaire was analyzed as $\%$ (percentile score) using the SoCQ quick scoring device [10] after the scores of the questions were averaged together.

\section{Research Results}

In this section, the results were presented to analyze teachers' interest in the developed criteria and to find ways to increase them and how to practice them.

\subsection{An Analysis of Interest in National Gifted Education Program Standards for Gifted Education Programs}

The results on gender interests are shown in [Table 3].

[Table 3] Attention by Gender (Percentile score)

\begin{tabular}{|c|c|c|c|c|c|c|c|c|}
\hline \multirow{2}{*}{\multicolumn{2}{|c|}{ Separate }} & \multicolumn{7}{|c|}{ By gender } \\
\hline & & One step & First & Two steps & Three steps & Three steps & Five steps & \\
\hline \multirow[b]{2}{*}{ Man } & Average & 23.40 & 22.38 & 22.66 & 22.98 & 22.52 & 22.35 & 23.42 \\
\hline & $\begin{array}{c}\text { Conversion } \\
\text { value }\end{array}$ & 99 & 80 & 80 & 85 & 43 & 55 & 77 \\
\hline \multirow[b]{2}{*}{ A woman } & Average & 24.32 & 23.37 & 23.98 & 24.12 & 23.58 & 23.60 & 24.55 \\
\hline & $\begin{array}{c}\text { Conversion } \\
\text { value }\end{array}$ & 99 & 84 & 83 & 88 & 48 & 64 & 84 \\
\hline
\end{tabular}

Looking at the relative strength of the level of interest according to the gender of gifted teachers, all of them showed high perceptual interest. For men, the order of results are perceptual, operational, personal, informative, cooperative, reinforcing, and consequential, followed by perceptual, operational, reinforcing, informative, personal, cooperative, and consequential. Overall, perceptual interest was high because of the burden of the national gifted program as a task. Nevertheless, the high level of operational and informational interest shows that gifted teachers want to know more about how the actual national gifted education program standards should be operated at the educational site, and know that they are closely related to gifted education.

Second, the level of interest by school level is shown in [Table 4].

[Table 4] Interest by School Level (Percentile score)

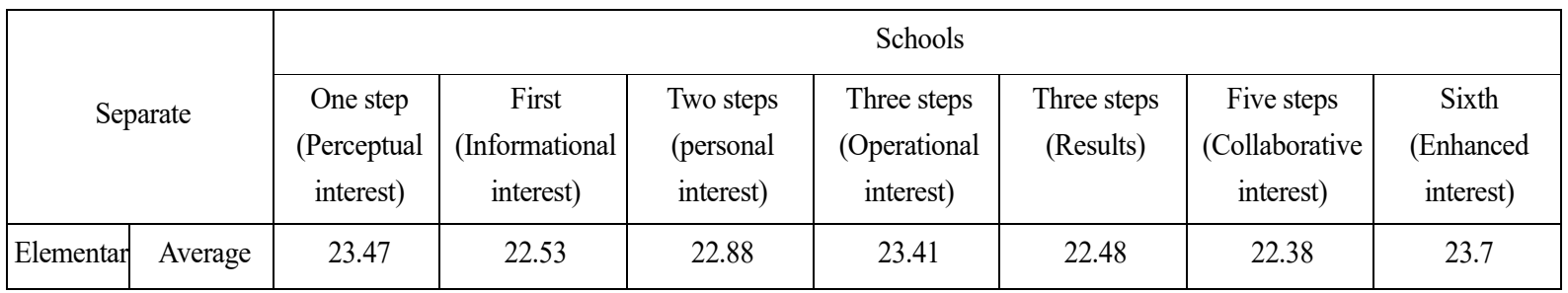




\begin{tabular}{|c|c|c|c|c|c|c|c|c|}
\hline $\begin{array}{c}\text { y School. } \\
\text { Teacher }\end{array}$ & $\begin{array}{c}\text { Conversion } \\
\text { value }\end{array}$ & 99 & 84 & 80 & 85 & 38 & 55 & 81 \\
\hline $\begin{array}{c}\text { Middle } \\
\text { school. }\end{array}$ & Average & 24.26 & 23 & 23.54 & 23.02 & 23.9 & 23.74 & 23.92 \\
\cline { 2 - 9 } Teachers & $\begin{array}{c}\text { Conversion } \\
\text { value }\end{array}$ & 99 & 84 & 83 & 85 & 48 & 64 & 81 \\
\hline
\end{tabular}

Looking at the average relative intensity of gifted teachers by level of interest according to school grades, both elementary and middle schools showed high perceptual interest. In the case of teachers participating in elementary school gifted education, perceptual interest, operational interest, informational interest, reinforcement interest, personal interest, cooperative interest, and consequential interest were shown.

Third, the interest level of each school's founding member is as shown in [Table 5].

[Table 5] Interest by Founder (Percentile score)

\begin{tabular}{|c|c|c|c|c|c|c|c|c|}
\hline \multicolumn{2}{|c|}{} & \multicolumn{7}{|c|}{ By establishment } \\
\cline { 2 - 9 } Separate & $\begin{array}{c}\text { One step } \\
\text { (Perceptual } \\
\text { interest) }\end{array}$ & $\begin{array}{c}\text { First } \\
\text { (Informational } \\
\text { interest) }\end{array}$ & $\begin{array}{c}\text { Two steps } \\
\text { (personal } \\
\text { interest) }\end{array}$ & $\begin{array}{c}\text { Three steps } \\
\text { (Operational } \\
\text { interest) }\end{array}$ & $\begin{array}{c}\text { Three steps } \\
\text { (Results) }\end{array}$ & $\begin{array}{c}\text { Five steps } \\
\text { (Collaborative } \\
\text { interest) }\end{array}$ & $\begin{array}{c}\text { Sixth } \\
\text { (Enhanced } \\
\text { interest) }\end{array}$ \\
\hline \multirow{2}{*}{$\begin{array}{c}\text { Public and } \\
\text { public }\end{array}$} & \begin{tabular}{c} 
Average \\
\cline { 2 - 9 }
\end{tabular} & 23.55 & 22.57 & 22.98 & 23.39 & 22.59 & 22.6 & 23.73 \\
\hline Palue & 99 & 84 & 80 & 85 & 43 & 59 & 81 \\
\cline { 2 - 9 } & $\begin{array}{c}\text { Conversion } \\
\text { Average }\end{array}$ & 24.33 & 23.23 & 23.4 & 22.8 & 24.2 & 23.4 & 23.8 \\
\hline
\end{tabular}

Looking at the average of relative intensity of each stage of interest according to each establishment, it was found that both national, public, and private schools had high perceptual interest. In the case of national and public, perceptual interest, operational interest, informational interest, reinforcement interest, personal interest, cooperative interest, and consequential interest were followed by perceptual interest, operational interest, informational interest, reinforcement interest, personal interest, cooperative interest.

Fourth, the results of interest in the total number of classes are shown in [Table 6].

[Table 6] Interest by Class Size (Percentile score)

\begin{tabular}{|c|c|c|c|c|c|c|c|c|}
\hline \multicolumn{2}{|c|}{} & \multicolumn{9}{|c|}{ Full class } \\
\cline { 2 - 9 } \multicolumn{2}{|c|}{ Separate } & $\begin{array}{c}\text { One step } \\
\text { (Perceptual } \\
\text { interest) }\end{array}$ & $\begin{array}{c}\text { First } \\
\text { (Informational } \\
\text { interest) }\end{array}$ & $\begin{array}{c}\text { Two steps } \\
\text { (personal } \\
\text { interest) }\end{array}$ & $\begin{array}{c}\text { Three steps } \\
\text { (Operational } \\
\text { interest) }\end{array}$ & $\begin{array}{c}\text { Three steps } \\
\text { (Results) }\end{array}$ & $\begin{array}{c}\text { Five steps } \\
\text { (Collaborative } \\
\text { interest) }\end{array}$ & $\begin{array}{c}\text { Sixth } \\
\text { (Enhanced } \\
\text { interest) }\end{array}$ \\
\hline \multirow{2}{*}{$\begin{array}{c}\text { The fifth } \\
\text { class }\end{array}$} & \begin{tabular}{c} 
Average \\
\cline { 2 - 9 } \\
Conversion \\
value
\end{tabular} & 23.9 & 21.7 & 22.6 & 23.45 & 21.8 & 23.09 & 24.09 \\
\hline 6-10 classes & Average & 22.93 & 22.26 & 22.33 & 22.4 & 22.8 & 21.46 & 22.93 \\
\cline { 2 - 10 } & $\begin{array}{c}\text { Conversion } \\
\text { value }\end{array}$ & 99 & 80 & 78 & 83 & 43 & 52 & 77 \\
\hline
\end{tabular}




\begin{tabular}{|c|c|c|c|c|c|c|c|c|}
\hline $\begin{array}{c}11-15 \\
\text { classes }\end{array}$ & Average & 23.82 & 22.72 & 22.94 & 23.08 & 23.04 & 22.7 & 23.46 \\
\cline { 2 - 9 } & $\begin{array}{c}\text { Conversion } \\
\text { value }\end{array}$ & 99 & 84 & 80 & 85 & 43 & 55 & 77 \\
\hline \multirow{2}{*}{$\begin{array}{c}\text { Morethan } 16 \\
\text { classes }\end{array}$} & \begin{tabular}{c} 
Average \\
\cline { 2 - 8 } \\
value
\end{tabular} & 23.67 & 22.79 & 23.20 & 23.50 & 22.84 & 22.84 & 23.94 \\
\hline
\end{tabular}

Looking at the mean of relative strength in stages of interest by overall grade, classes 5 or below were followed by perceptual, operational, reinforcement, personal, information, collaborative, and consequential, and 6-10 classes were presented in order of perceptual, operational, personal, reinforcement, collaborative, 11-15 Classes were presented in the order of perceptual, operational, informational, personal, reinforcement, cooperative, and consequential, with classes 16 or higher in the order of perceptual, operational, informational, reinforcement, personal, collaborative, and consequential.

Fifth, the degree of interest for each teaching career is shown in [Table 7].

[Table 7] Interest by Teaching Experience (Percentile Score)

\begin{tabular}{|c|c|c|c|c|c|c|c|c|}
\hline \multirow{2}{*}{\multicolumn{2}{|c|}{ Separate }} & \multicolumn{7}{|c|}{ By orthographic force } \\
\hline & & \multirow{2}{*}{ 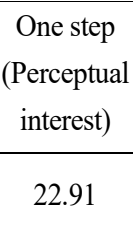 } & \multirow{2}{*}{$\begin{array}{c}\text { First } \\
\text { (Informational } \\
\text { interest) }\end{array}$} & \multirow{2}{*}{$\begin{array}{c}\text { Two steps } \\
\text { (personal } \\
\text { interest) }\end{array}$} & \multirow{2}{*}{ 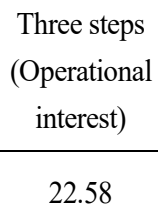 } & \multirow{2}{*}{$\begin{array}{c}\text { Three steps } \\
\text { (Results) }\end{array}$} & \multirow{2}{*}{$\begin{array}{c}\begin{array}{c}\text { Five steps } \\
\text { (Collaborative } \\
\text { interest) }\end{array} \\
21.58\end{array}$} & \multirow{2}{*}{$\begin{array}{c}\begin{array}{c}\text { Sixth } \\
\text { (Enhanced } \\
\text { interest) }\end{array} \\
22.91\end{array}$} \\
\hline For four & Average & & & & & & & \\
\hline years & $\begin{array}{c}\text { Conversion } \\
\text { value }\end{array}$ & 99 & 80 & 80 & 85 & 43 & 55 & 77 \\
\hline \multirow[b]{2}{*}{$5-9$ years } & Average & 24 & 23.31 & 22.12 & 23.18 & 21.87 & 21.75 & 23.62 \\
\hline & $\begin{array}{c}\text { Conversion } \\
\text { value }\end{array}$ & 99 & 84 & 78 & 85 & 38 & 55 & 81 \\
\hline \multirow[b]{2}{*}{ In $10-14$} & Average & 23.9 & 22.87 & 23.21 & 23.63 & 22.96 & 22.72 & 23.87 \\
\hline & $\begin{array}{c}\text { Conversion } \\
\text { value }\end{array}$ & 99 & 84 & 80 & 88 & 43 & 59 & 81 \\
\hline \multirow[b]{2}{*}{ In $15-19$} & Average & 23.61 & 22.77 & 23.19 & 22.31 & 23 & 22.87 & 23.77 \\
\hline & $\begin{array}{c}\text { Conversion } \\
\text { value }\end{array}$ & 99 & 84 & 80 & 83 & 43 & 59 & 81 \\
\hline \multirow{2}{*}{$\begin{array}{c}\text { For more } \\
\text { than } 20 \\
\text { years }\end{array}$} & Average & 23.91 & 21.91 & 22.75 & 23.33 & 23 & 23.5 & 24.16 \\
\hline & $\begin{array}{c}\text { Conversion } \\
\text { value }\end{array}$ & 99 & 80 & 80 & 85 & 43 & 64 & 81 \\
\hline
\end{tabular}

Looking at the mean of relative strength in stages of interest by teaching career, 0-4 years followed the perceptual, operational, personal, informational, reinforcement, collaborative, and consequential, and 5-9 years followed the perceptual, operational, informative, personal, collaborative, and consequential. Perceptual interest, operational interest, reinforcement interest, personal interest, informational interest, cooperative interest, and consequential interest were shown.

Sixth, the degree of interest by academic background is shown in [Table 8]. 
[Table 8] Depth by academic background (Percentile score)

\begin{tabular}{|c|c|c|c|c|c|c|c|c|}
\hline \multicolumn{2}{|c|}{} & \multicolumn{9}{|c|}{ By academic force } \\
\cline { 2 - 9 } \multicolumn{2}{|c|}{ Separate } & $\begin{array}{c}\text { One step } \\
\text { (Perceptual } \\
\text { interest) }\end{array}$ & $\begin{array}{c}\text { First } \\
\text { (Informational } \\
\text { interest) }\end{array}$ & $\begin{array}{c}\text { Two steps } \\
\text { (personal } \\
\text { interest) }\end{array}$ & $\begin{array}{c}\text { Three steps } \\
\text { (Operational } \\
\text { interest) }\end{array}$ & $\begin{array}{c}\text { Three steps } \\
\text { (Results) }\end{array}$ & $\begin{array}{c}\text { Five steps } \\
\text { (Collaborative } \\
\text { interest) }\end{array}$ & $\begin{array}{c}\text { Sixth } \\
\text { (Enhanced } \\
\text { interest) }\end{array}$ \\
\hline \multirow{2}{*}{ Academy } & Average & 23.16 & 22.27 & 22.54 & 22.79 & 22.43 & 22.10 & 23.14 \\
\cline { 2 - 10 } & $\begin{array}{c}\text { Conversio } \\
\text { n value }\end{array}$ & 99 & 80 & 80 & 85 & 38 & 55 & 77 \\
\hline $\begin{array}{c}\text { Seok Sai- } \\
\text { sang }\end{array}$ & \begin{tabular}{c} 
Average \\
\cline { 2 - 9 } \\
Conversio \\
n value
\end{tabular} & 24.33 & 23.20 & 23.70 & 24 & 23.36 & 23.53 & 24.55 \\
\hline
\end{tabular}

The average of relative strength in stages of interest by academic background was shown in the order of perceptual interest, operational interest, informational interest, personal interest, reinforcement interest, and cooperative interest for master's or higher.

Seventh, the degree of interest by position is shown in [Table 9].

[Table 9] Depth by Position (Percentile score)

\begin{tabular}{|c|c|c|c|c|c|c|c|c|}
\hline \multicolumn{2}{|c|}{} & \multicolumn{9}{|c|}{ By title } \\
\cline { 2 - 9 } \multicolumn{2}{|c|}{ Separate } & $\begin{array}{c}\text { One step } \\
\text { (Perceptual } \\
\text { interest) }\end{array}$ & $\begin{array}{c}\text { First } \\
\text { (Informational } \\
\text { interest) }\end{array}$ & $\begin{array}{c}\text { Two steps } \\
\text { (personal } \\
\text { interest) }\end{array}$ & $\begin{array}{c}\text { Three steps } \\
\text { (Operational } \\
\text { interest) }\end{array}$ & $\begin{array}{c}\text { Three steps } \\
\text { (Results) }\end{array}$ & $\begin{array}{c}\text { Five steps } \\
\text { (Collaborative } \\
\text { interest) }\end{array}$ & $\begin{array}{c}\text { Sixth } \\
\text { (Enhanced } \\
\text { interest) }\end{array}$ \\
\hline \begin{tabular}{c} 
Principles \\
\cline { 2 - 10 }
\end{tabular} & $\begin{array}{c}\text { Average } \\
\text { Conversion } \\
\text { value }\end{array}$ & 23.42 & 22.35 & 22.85 & 22.85 & 22.7 & 22.66 & 23.35 \\
\hline $\begin{array}{c}\text { A } \\
\text { vocational } \\
\text { teacher }\end{array}$ & $\begin{array}{c}\text { Average } \\
\text { Conversion } \\
\text { value }\end{array}$ & 24.4 & 23.64 & 23.62 & 24.7 & 23.24 & 22.88 & 24.96 \\
\hline
\end{tabular}

Looking at the mean of relative strength in stages of interest by position, lay teachers showed perceptual interest, operational interest, reinforcement, personal interest, informational interest, collaborative interest, vocational interest, information, operational interest, personal interest, and cooperative interest. Eighth, the level of interest in each field is shown in [Table 10].

[Table 10] Depth by Discipline (Percentile score)

\begin{tabular}{|c|c|c|c|c|c|c|c|c|}
\hline \multicolumn{2}{|c|}{} & \multicolumn{9}{|c|}{ By field } \\
\cline { 2 - 9 } Separate & $\begin{array}{c}\text { One step } \\
\text { (Perceptual } \\
\text { interest) }\end{array}$ & $\begin{array}{c}\text { First } \\
\text { (Informational } \\
\text { interest) }\end{array}$ & $\begin{array}{c}\text { Two steps } \\
\text { (personal } \\
\text { interest) }\end{array}$ & $\begin{array}{c}\text { Three steps } \\
\text { (Operational } \\
\text { interest) }\end{array}$ & $\begin{array}{c}\text { Three steps } \\
\text { (Results) }\end{array}$ & $\begin{array}{c}\text { Five steps } \\
\text { (Collaborative } \\
\text { interest) }\end{array}$ & $\begin{array}{c}\text { Sixth } \\
\text { (Enhanced } \\
\text { interest) }\end{array}$ \\
\hline Science & $\begin{array}{c}\text { Average } \\
\text { Conversion } \\
\text { value }\end{array}$ & 23.62 & 22.56 & 22.92 & 23.09 & 22.81 & 22.69 & 23.53 \\
\hline Math & Average & 23.83 & 23.06 & 23.45 & 24.11 & 22.90 & 22.81 & 89 \\
\hline
\end{tabular}




\begin{tabular}{|c|c|c|c|c|c|c|c|c|}
\hline $\begin{array}{c}\text { Conversion } \\
\text { value }\end{array}$ & 99 & 84 & 80 & 88 & 43 & 59 & 84 \\
\hline
\end{tabular}

Looking at the mean of relative intensity by stages of interest by discipline, science showed perceptual interest, operational interest, informational interest, reinforcement, personal interest, cooperative interest, consequential interest, mathematics in the order of perceptual interest, operational interest, information interest, personal interest, and cooperative interest.

Ninth, the overall interest is shown in [Table 11].

[Table 11] Overall Depth (Percentile score)

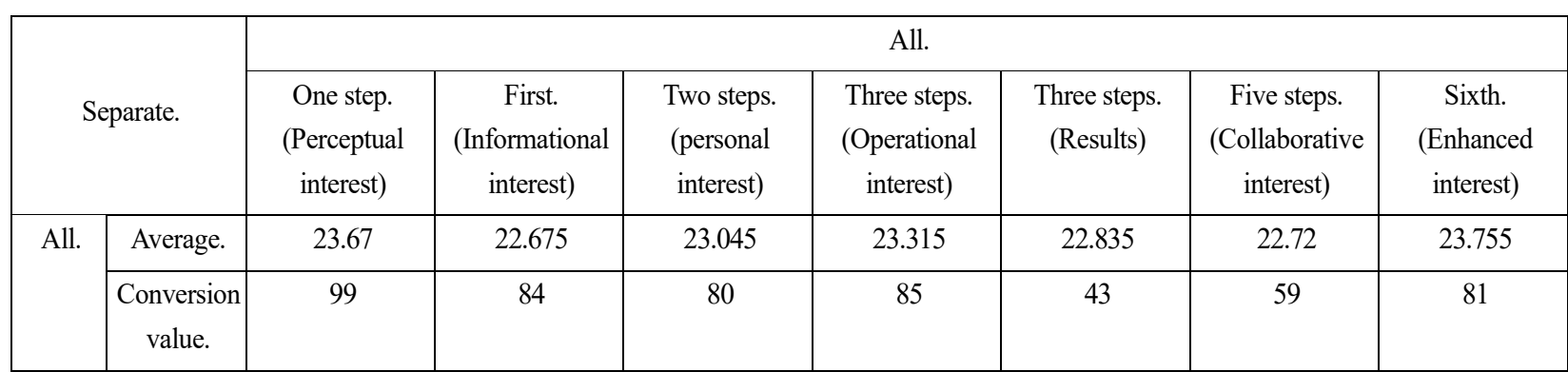

The overall mean of the relative strength of the step-by-step of interest according to the discipline, for interest, perceptual, operational, informational, reinforcement, personal, cooperative, and consequential is shown below [Fig. 1].

\section{Total}

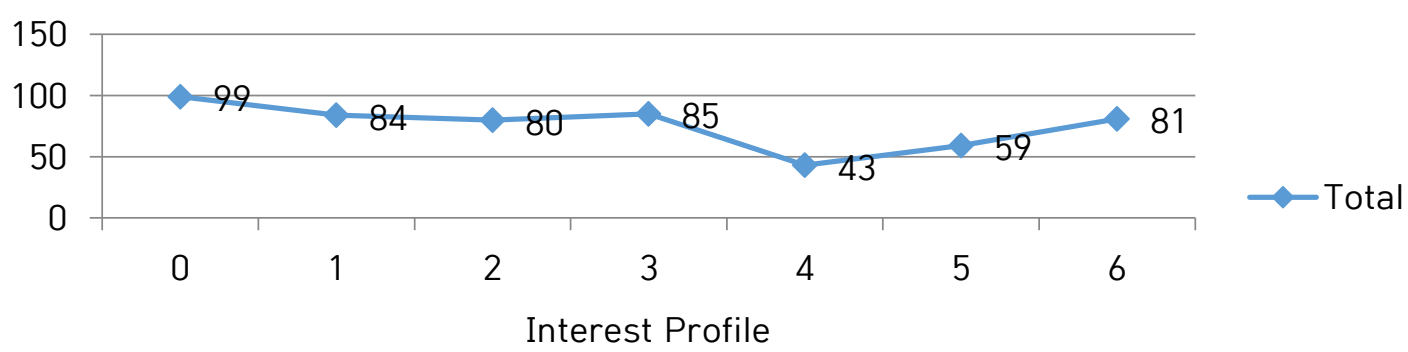

[Fig. 1] Interest Profile

\subsection{Differences in Interest in National Gifted Education Programs by Background Variable}

In the analysis of differences in interest, the independent sample t-test by gender, school grade, founder, and teaching experience showed significant differences in cooperative interest by academic background, strengthening interest by academic background, and operating interest by position. In addition, one-way Anova was conducted to examine whether there are differences in interest levels according to variables for analysis of differences in class or class size, depending on the number of teaching experiences in groups. As a result of one-way random analysis, no significant differences were found depending on teaching experience, number of classes, or class size. Details are as follows.

[Table 12] Gender t-test Results

\begin{tabular}{|c|c|c|c|c|c|c|}
\hline Separate & Group & $\mathrm{N}$ & $\mathrm{M}$ & $\mathrm{SD}$ & $\mathrm{t}$ & $\mathrm{p}$ \\
\hline One step & Man. & 142 & 23.4014 & 4.89182 & -1.240 & .216 \\
\hline
\end{tabular}




\begin{tabular}{|c|c|c|c|c|c|c|}
\hline $\begin{array}{l}\text { (Perceptual } \\
\text { interest) }\end{array}$ & A woman. & 58 & 24.3276 & 4.53984 & & \\
\hline \multirow{2}{*}{$\begin{array}{c}\text { First } \\
\text { (Informational } \\
\text { interest) }\end{array}$} & Man. & 142 & 22.3873 & 4.21219 & \multirow{2}{*}{-1.495} & \multirow{2}{*}{.137} \\
\hline & A woman. & 58 & 23.3793 & 4.37227 & & \\
\hline \multirow{2}{*}{$\begin{array}{c}\text { Two steps } \\
\text { (personal interest) }\end{array}$} & Man. & 142 & 22.6620 & 4.62937 & \multirow{2}{*}{-1.807} & \multirow{2}{*}{.072} \\
\hline & A woman. & 58 & 23.9828 & 4.83587 & & \\
\hline \multirow{2}{*}{$\begin{array}{l}\text { Three steps } \\
\text { (Operational } \\
\text { interest) }\end{array}$} & Man. & 142 & 22.9859 & 4.75944 & \multirow{2}{*}{-1.508} & \multirow{2}{*}{.133} \\
\hline & A woman. & 58 & 24.1207 & 5.00203 & & \\
\hline \multirow{2}{*}{$\begin{array}{l}\text { Three steps } \\
\text { (Results) }\end{array}$} & Man. & 142 & 22.5282 & 4.92165 & \multirow{2}{*}{-1.373} & \multirow{2}{*}{.171} \\
\hline & A woman. & 58 & 23.5862 & 5.00188 & & \\
\hline \multirow{2}{*}{$\begin{array}{c}\text { Five steps } \\
\text { (Collaborative } \\
\text { interest) }\end{array}$} & Man. & 142 & 22.3592 & 4.96990 & \multirow{2}{*}{-1.635} & \multirow{2}{*}{.104} \\
\hline & A woman. & 58 & 23.6034 & 4.66017 & & \\
\hline \multirow{2}{*}{$\begin{array}{c}\text { Sixth } \\
\text { (Enhanced interest) }\end{array}$} & Man. & 142 & 22.3592 & 4.96990 & \multirow{2}{*}{-1.506} & \multirow{2}{*}{.134} \\
\hline & A woman. & 58 & 23.6034 & 4.66017 & & \\
\hline
\end{tabular}

${ }^{*} \mathrm{p}<.05,{ }^{* *} \mathrm{p}<.01,{ }^{* * *} \mathrm{p}<.001$

[Table 13] T-test Results by School Level

\begin{tabular}{|c|c|c|c|c|c|c|}
\hline Separate & Group & $\mathrm{N}$ & M & SD & $\mathrm{t}$ & $\mathrm{p}$ \\
\hline \multirow{2}{*}{$\begin{array}{l}\text { One step } \\
\text { (Perceptual } \\
\text { interest) }\end{array}$} & $\begin{array}{l}\text { Participation teachers in } \\
\text { elementary school }\end{array}$ & 150 & 23.4733 & 4.80170 & \multirow{2}{*}{-1.004} & \multirow{2}{*}{.317} \\
\hline & $\begin{array}{l}\text { Participation teachers in } \\
\text { middle school }\end{array}$ & 50 & 24.2600 & 4.79289 & & \\
\hline \multirow{2}{*}{$\begin{array}{c}\text { First } \\
\text { (Informational } \\
\text { interest) }\end{array}$} & $\begin{array}{l}\text { Participation teachers in } \\
\text { elementary school }\end{array}$ & 150 & 22.5667 & 4.25751 & \multirow{2}{*}{-.620} & \multirow{2}{*}{.536} \\
\hline & $\begin{array}{l}\text { Participation teachers in } \\
\text { middle school }\end{array}$ & 50 & 23.0000 & 4.34248 & & \\
\hline \multirow{2}{*}{$\begin{array}{c}\text { Two steps } \\
\text { (personal interest) }\end{array}$} & $\begin{array}{l}\text { Participation teachers in } \\
\text { elementary school }\end{array}$ & 150 & 22.8800 & 4.70744 & \multirow{2}{*}{-.856} & \multirow{2}{*}{.393} \\
\hline & $\begin{array}{l}\text { Participation teachers in } \\
\text { middle school }\end{array}$ & 50 & 23.5400 & 4.75613 & & \\
\hline \multirow{2}{*}{$\begin{array}{l}\text { Three steps } \\
\text { (Operational } \\
\text { interest) }\end{array}$} & $\begin{array}{l}\text { Participation teachers in } \\
\text { elementary school }\end{array}$ & 150 & 23.4133 & 4.84136 & \multirow{2}{*}{.496} & \multirow{2}{*}{.620} \\
\hline & $\begin{array}{l}\text { Participation teachers in } \\
\text { middle school }\end{array}$ & 50 & 23.0200 & 4.89685 & & \\
\hline \multirow{2}{*}{$\begin{array}{l}\text { Three steps } \\
\text { (Results) }\end{array}$} & $\begin{array}{l}\text { Participation teachers in } \\
\text { elementary school }\end{array}$ & 150 & 22.4800 & 4.95851 & \multirow{2}{*}{-1.764} & \multirow{2}{*}{.079} \\
\hline & $\begin{array}{l}\text { Participation teachers in } \\
\text { middle school. }\end{array}$ & 50 & 23.9000 & 4.84136 & & \\
\hline $\begin{array}{l}\text { Five steps. } \\
\text { (Collaborative }\end{array}$ & $\begin{array}{l}\text { Participation teachers in } \\
\text { elementary school. }\end{array}$ & 150 & 22.3800 & 4.89853 & -1.707 & .089 \\
\hline
\end{tabular}




\begin{tabular}{|c|c|c|c|c|c|c|}
\hline interest) & $\begin{array}{l}\text { Participation teachers in } \\
\text { middle school. }\end{array}$ & 50 & 23.7400 & 4.82261 & & \\
\hline \multirow{2}{*}{$\begin{array}{c}\text { Sixth. } \\
\text { (Enhanced } \\
\text { interest) }\end{array}$} & $\begin{array}{l}\text { Participation teachers in } \\
\text { elementary school. }\end{array}$ & 150 & 23.7000 & 4.86702 & \multirow{2}{*}{-.280} & \multirow{2}{*}{.780} \\
\hline & $\begin{array}{l}\text { Participation teachers in } \\
\text { middle school. }\end{array}$ & 50 & 23.9200 & 4.61957 & & \\
\hline
\end{tabular}

${ }^{*} \mathrm{p}<.05,{ }^{* *} \mathrm{p}<.01, * * * \mathrm{p}<.001$

[Table 14] T-test Results by Establishment Subject

\begin{tabular}{|c|c|c|c|c|c|c|}
\hline Separate & Group & $\mathrm{N}$ & M & SD & $\mathrm{t}$ & $\mathrm{p}$ \\
\hline \multirow{2}{*}{$\begin{array}{c}\text { One step } \\
\text { (Perceptual interest) }\end{array}$} & Public public relations & 170 & 23.5529 & 4.80139 & \multirow{2}{*}{-.820} & \multirow{2}{*}{.413} \\
\hline & Private & 30 & 24.3333 & 4.81616 & & \\
\hline \multirow{2}{*}{$\begin{array}{c}\text { First } \\
\text { (Informational } \\
\text { interest) }\end{array}$} & Public public relations & 170 & 22.5765 & 4.22833 & \multirow[t]{2}{*}{-.776} & \multirow[t]{2}{*}{.439} \\
\hline & Private & 30 & 23.2333 & 4.54619 & & \\
\hline \multirow{2}{*}{$\begin{array}{c}\text { Two steps } \\
\text { (personal interest) }\end{array}$} & Public public relations & 170 & 22.9824 & 4.71994 & \multirow{2}{*}{-.446} & \multirow{2}{*}{.656} \\
\hline & Private & 30 & 23.4000 & 4.76047 & & \\
\hline \multirow{2}{*}{$\begin{array}{c}\text { Three steps } \\
\text { (Operational interest) }\end{array}$} & Public public relations & 170 & 23.3941 & 4.81036 & \multirow{2}{*}{.549} & \multirow{2}{*}{.584} \\
\hline & Private & 30 & 22.8667 & 5.10398 & & \\
\hline \multirow{2}{*}{$\begin{array}{l}\text { Three steps } \\
\text { (Results) }\end{array}$} & Public public relations & 170 & 22.5941 & 4.93568 & \multirow{2}{*}{-1.643} & \multirow{2}{*}{.102} \\
\hline & Private & 30 & 24.2000 & 4.92985 & & \\
\hline \multirow{2}{*}{$\begin{array}{c}\text { Five steps } \\
\text { (Collaborative } \\
\text { interest) }\end{array}$} & Public public relations & 170 & 22.6000 & 4.90188 & \multirow[t]{2}{*}{-.823} & \multirow[t]{2}{*}{.411} \\
\hline & Private & 30 & 23.4000 & 4.93824 & & \\
\hline \multirow{2}{*}{$\begin{array}{c}\text { Sixth } \\
\text { (Enhanced interest) }\end{array}$} & Public public relations & 170 & 23.7353 & 4.83031 & \multirow{2}{*}{-.138} & \multirow{2}{*}{.890} \\
\hline & Private & 30 & 23.8667 & 4.67372 & & \\
\hline
\end{tabular}

${ }^{*} \mathrm{p}<.05,{ }^{* *} \mathrm{p}<.01,{ }^{* * *} \mathrm{p}<.001$

[Table 15] T-test Results by Degree

\begin{tabular}{|c|c|c|c|c|c|c|}
\hline Separate & Group & $\mathrm{N}$ & M & SD & $\mathrm{t}$ & $\mathrm{p}$ \\
\hline \multirow{2}{*}{$\begin{array}{c}\text { One step } \\
\text { (Perceptual interest) }\end{array}$} & Academy & 114 & 23.1667 & 4.78921 & \multirow{2}{*}{-1.716} & \multirow{2}{*}{.088} \\
\hline & The master & 86 & 24.3372 & 4.75917 & & \\
\hline \multirow{2}{*}{$\begin{array}{c}\text { First } \\
\text { (Informational } \\
\text { interest) }\end{array}$} & Academy & 114 & 22.2719 & 4.20132 & \multirow{2}{*}{-1.542} & \multirow{2}{*}{.125} \\
\hline & The master & 86 & 23.2093 & 4.33078 & & \\
\hline \multirow{2}{*}{$\begin{array}{c}\text { Two steps } \\
\text { (personal interest) }\end{array}$} & Academy & 114 & 22.5439 & 4.66988 & \multirow{2}{*}{-1.739} & \multirow{2}{*}{.084} \\
\hline & The master & 86 & 23.7093 & 4.72258 & & \\
\hline Three steps & Academy & 114 & 22.7982 & 4.81917 & -1.745 & .083 \\
\hline
\end{tabular}




\begin{tabular}{|c|c|c|c|c|c|c|}
\hline (Operational interest) & The master & 86 & 24.0000 & 4.82396 & & \\
\hline \multirow{2}{*}{$\begin{array}{l}\text { Three steps } \\
\text { (Results) }\end{array}$} & Academy & 114 & 22.4386 & 4.89181 & \multirow{2}{*}{-1.305} & \multirow{2}{*}{.194} \\
\hline & The master & 86 & 23.3605 & 5.01976 & & \\
\hline \multirow{2}{*}{$\begin{array}{c}\text { Five steps } \\
\text { (Collaborative } \\
\text { interest) }\end{array}$} & Academy & 114 & 22.1053 & 4.90686 & \multirow{2}{*}{-2.058} & \multirow{2}{*}{$.041^{\prime}$} \\
\hline & The master & 86 & 23.5349 & 4.80612 & & \\
\hline \multirow{2}{*}{$\begin{array}{c}\text { Sixth } \\
\text { (Enhanced interest) }\end{array}$} & Academy & 114 & 23.1491 & 4.82844 & \multirow{2}{*}{.45222} & \multirow{2}{*}{$.039^{\circ}$} \\
\hline & The master & 86 & 24.5581 & 4.65922 & & \\
\hline
\end{tabular}

${ }^{*} \mathrm{p}<.05,{ }^{* *} \mathrm{p}<.01,{ }^{* * *} \mathrm{p}<.001$

[Table 16] t-test Results by Position

\begin{tabular}{|c|c|c|c|c|c|c|}
\hline Separate & Group & $\mathrm{N}$ & M & SD & $\mathrm{t}$ & $\mathrm{p}$ \\
\hline \multirow{2}{*}{$\begin{array}{c}\text { One step } \\
\text { (Perceptual interest) }\end{array}$} & Principles & 150 & 23.4267 & 4.96342 & \multirow{2}{*}{-1.244} & \multirow{2}{*}{.215} \\
\hline & A vocational teacher & 50 & 24.4000 & 4.23301 & & \\
\hline \multirow{2}{*}{$\begin{array}{c}\text { First } \\
\text { (Informational } \\
\text { interest) }\end{array}$} & Principles & 150 & 22.3533 & 4.39514 & \multirow{2}{*}{-1.856} & \multirow{2}{*}{.065} \\
\hline & A vocational teacher & 50 & 23.6400 & 3.75668 & & \\
\hline \multirow{2}{*}{$\begin{array}{c}\text { Two steps } \\
\text { (personal interest) }\end{array}$} & Principles & 150 & 22.8533 & 4.80127 & \multirow{2}{*}{-.995} & \multirow{2}{*}{.321} \\
\hline & A vocational teacher & 50 & 23.6200 & 4.44876 & & \\
\hline \multirow{2}{*}{$\begin{array}{c}\text { Three steps } \\
\text { (Operational interest) }\end{array}$} & Principles & 150 & 22.8533 & 4.90362 & \multirow{2}{*}{-2.360} & \multirow{2}{*}{$.019 *$} \\
\hline & A vocational teacher & 50 & 24.7000 & 4.43203 & & \\
\hline \multirow{2}{*}{$\begin{array}{c}\text { Three steps } \\
\text { (Results) }\end{array}$} & Principles & 150 & 22.7000 & 5.01106 & \multirow{2}{*}{-.666} & \multirow{2}{*}{.506} \\
\hline & A vocational teacher & 50 & 23.2400 & 4.81308 & & \\
\hline \multirow{2}{*}{$\begin{array}{c}\text { Five steps } \\
\text { (Collaborative } \\
\text { interest) }\end{array}$} & Principles & 150 & 22.6667 & 5.01764 & \multirow{2}{*}{-.266} & \multirow{2}{*}{.791} \\
\hline & A vocational teacher & 50 & 22.8800 & 4.58765 & & \\
\hline \multirow{2}{*}{$\begin{array}{c}\text { Sixth } \\
\text { (Enhanced interest) }\end{array}$} & Principles & 150 & 23.3533 & 4.83296 & \multirow{2}{*}{-2.068} & \multirow{2}{*}{.040} \\
\hline & A vocational teacher & 50 & 24.9600 & 4.51736 & & \\
\hline
\end{tabular}

${ }^{*} \mathrm{p}<.05,{ }^{* *} \mathrm{p}<.01,{ }^{* * *} \mathrm{p}<.001$

[Table 17] T-test results by Field

\begin{tabular}{|c|c|c|c|c|c|c|}
\hline Separate & Group & $\mathrm{N}$ & M & SD & $\mathrm{t}$ & $\mathrm{p}$ \\
\hline \multirow{2}{*}{$\begin{array}{l}\text { One step } \\
\text { (Perceptual } \\
\text { interest) }\end{array}$} & Science & 157 & 23.6242 & 4.79505 & \multirow{2}{*}{-.257} & \multirow{2}{*}{.797} \\
\hline & Math & 43 & 23.8372 & 4.86939 & & \\
\hline \multirow{2}{*}{$\begin{array}{c}\text { First } \\
\text { (Informational } \\
\text { interest) }\end{array}$} & Science & 157 & 22.5669 & 4.35342 & \multirow{2}{*}{-.683} & \multirow{2}{*}{.495} \\
\hline & Math & 43 & 23.0698 & 3.98447 & & \\
\hline
\end{tabular}




\begin{tabular}{|c|c|c|c|c|c|c|}
\hline Two steps & Science & 157 & 22.9299 & 4.82530 & \multirow{2}{*}{-.658} & \multirow{2}{*}{.511} \\
\hline (personal interest) & Math & 43 & 23.4651 & 4.32242 & & \\
\hline \multirow{2}{*}{$\begin{array}{l}\text { Three steps } \\
\text { (Operational } \\
\text { interest) }\end{array}$} & Science & 157 & 23.0955 & 4.77008 & \multirow{2}{*}{-1.225} & \multirow{2}{*}{.222} \\
\hline & Math & 43 & 24.1163 & 5.09065 & & \\
\hline \multirow{2}{*}{$\begin{array}{c}\text { Three steps } \\
\text { (Results) }\end{array}$} & Science & 157 & 22.8153 & 5.07990 & \multirow{2}{*}{-.107} & \multirow{2}{*}{.915} \\
\hline & Math & 43 & 22.9070 & 4.52934 & & \\
\hline \multirow{2}{*}{$\begin{array}{c}\text { Five steps } \\
\text { (Collaborative } \\
\text { interest) }\end{array}$} & Science & 157 & 22.6943 & 5.08853 & \multirow{2}{*}{-.141} & \multirow{2}{*}{.888} \\
\hline & Math & 43 & 22.8140 & 4.21028 & & \\
\hline \multirow{2}{*}{$\begin{array}{c}\text { Sixth } \\
\text { (Enhanced } \\
\text { interest) }\end{array}$} & Science & 157 & 23.5350 & 4.69250 & \multirow{2}{*}{-1.241} & \multirow{2}{*}{.216} \\
\hline & Math & 43 & 24.5581 & 5.13300 & & \\
\hline
\end{tabular}

${ }^{*} \mathrm{p}<.05,{ }^{* *} \mathrm{p}<.01,{ }^{* * *} \mathrm{p}<.001$

[Table 18] Results of One-way Random Analysis on the Interest Level of Each Class

\begin{tabular}{|c|c|c|c|c|c|c|}
\hline \multirow{2}{*}{ Area } & Source & \multirow{2}{*}{ SS } & \multirow{2}{*}{ DF } & \multirow{2}{*}{ MS } & F- & \multirow{2}{*}{$\operatorname{Pr}>\mathrm{F}$} \\
\hline & Class numbers & & & & Value & \\
\hline \multirow{3}{*}{$\begin{array}{c}\text { One step } \\
\text { (Perceptual interest) }\end{array}$} & Group-liver & 9.901 & 3 & 3.300 & .141 & .935 \\
\hline & Group-mine & 4574.319 & 196 & 23.338 & & \\
\hline & Total & 4584.220 & 199 & & & \\
\hline \multirow{3}{*}{$\begin{array}{c}\text { First } \\
\text { (Informational } \\
\text { interest) }\end{array}$} & Group-liver & 14.131 & 3 & 4.710 & .255 & .858 \\
\hline & Group-mine & 3617.744 & 196 & 18.458 & & \\
\hline & Total & 3631.875 & 199 & & & \\
\hline \multirow{3}{*}{$\begin{array}{c}\text { Two steps } \\
\text { (personal interest) }\end{array}$} & Group-liver & 13.348 & 3 & 4.449 & .198 & .898 \\
\hline & Group-mine & 4413.247 & 196 & 22.517 & & \\
\hline & Total & 4426.595 & 199 & & & \\
\hline \multirow{3}{*}{$\begin{array}{l}\text { Three steps } \\
\text { (Operational } \\
\text { interest) }\end{array}$} & Group-liver & 20.156 & 3 & 6.719 & .283 & .838 \\
\hline & Group-mine & 4652.999 & 196 & 23.740 & & \\
\hline & Total & 4673.155 & 199 & & & \\
\hline \multirow{3}{*}{$\begin{array}{l}\text { Three steps } \\
\text { (Results) }\end{array}$} & Group-liver & 13.510 & 3 & 4.503 & .181 & .909 \\
\hline & Group-mine & 4874.045 & 196 & 24.868 & & \\
\hline & Total & 4887.555 & 199 & & & \\
\hline \multirow{3}{*}{$\begin{array}{c}\text { Five steps } \\
\text { (Collaborative } \\
\text { interest) }\end{array}$} & Group-liver & 27.089 & 3 & 9.030 & .372 & .773 \\
\hline & Group-mine & 4757.231 & 196 & 24.272 & & \\
\hline & Total & 4784.320 & 199 & & & \\
\hline \multirow{3}{*}{$\begin{array}{c}\text { Sixth } \\
\text { (Enhanced interest) }\end{array}$} & Group-liver & 20.128 & 3 & 6.709 & .289 & .834 \\
\hline & Group-mine & 4556.867 & 196 & 23.249 & & \\
\hline & Total & 4576.995 & 199 & & & \\
\hline
\end{tabular}

${ }^{*} \mathrm{p}<.05,{ }^{* *} \mathrm{p}<.01,{ }^{* * *} \mathrm{p}<.001$ 
[Table 19] Results of One-way Random Analysis on the Degree of Interest by Teaching Experience

\begin{tabular}{|c|c|c|c|c|c|c|}
\hline \multirow[b]{2}{*}{ Area } & Source & \multirow[b]{2}{*}{ SS } & \multirow[b]{2}{*}{ DF } & \multirow[b]{2}{*}{ MS } & \multirow{2}{*}{$\begin{array}{c}\text { F- } \\
\text { Value }\end{array}$} & \multirow[b]{2}{*}{$\operatorname{Pr}>\mathrm{F}$} \\
\hline & $\begin{array}{c}\text { By orthographic } \\
\text { force }\end{array}$ & & & & & \\
\hline \multirow{3}{*}{$\begin{array}{c}\text { One step } \\
\text { (Perceptual interest) }\end{array}$} & Group-liver & 11.565 & 4 & 2.891 & .123 & .974 \\
\hline & Group-mine & 4572.655 & 195 & 23.450 & & \\
\hline & Total & 4584.220 & 199 & & & \\
\hline \multirow{3}{*}{$\begin{array}{c}\text { First } \\
\text { (Informational interest) }\end{array}$} & Group-liver & 12.961 & 4 & 3.240 & .175 & .951 \\
\hline & Group-mine & 3618.914 & 195 & 18.559 & & \\
\hline & Total & 3631.875 & 199 & & & \\
\hline \multirow{3}{*}{$\begin{array}{c}\text { Two steps } \\
\text { (personal interest) }\end{array}$} & Group-liver & 22.001 & 4 & 5.500 & 244 & .913 \\
\hline & Group-mine & 4404.594 & 195 & 22.588 & & \\
\hline & Total & 4426.595 & 199 & & & \\
\hline \multirow{3}{*}{$\begin{array}{c}\text { Three steps } \\
\text { (Operational interest) }\end{array}$} & Group-liver & 10.096 & 4 & 2.524 & . 106 & .980 \\
\hline & Group-mine & 4663.059 & 195 & 23.913 & & \\
\hline & Total & 4673.155 & 199 & & & \\
\hline \multirow{3}{*}{$\begin{array}{l}\text { Three steps } \\
\text { (Results) }\end{array}$} & Group-liver & 27.169 & 4 & 6.792 & .273 & .895 \\
\hline & Group-mine & 4860.386 & 195 & 24.925 & & \\
\hline & Total & 4887.555 & 199 & & & \\
\hline \multirow{3}{*}{$\begin{array}{c}\text { Five steps } \\
\text { (Collaborative interest) }\end{array}$} & Group-liver & 40.874 & 4 & 10.218 & .420 & .794 \\
\hline & Group-mine & 4743.446 & 195 & 24.325 & & \\
\hline & Total & 4784.320 & 199 & & & \\
\hline \multirow{3}{*}{$\begin{array}{c}\text { Sixth } \\
\text { (Enhanced interest) }\end{array}$} & Group-liver & 11.320 & 4 & 2.830 & .121 & .975 \\
\hline & Group-mine & 4565.675 & 195 & 23.414 & & \\
\hline & Total & 4576.995 & 199 & & & \\
\hline
\end{tabular}

Independent sample t-test and one-way analysis of variance (one-way Anova) were conducted to see if there were any differences in interest levels depending on the variables. As a result, collaborative interest by academic background $(\mathrm{p}<.05)$ to $\mathrm{F}=-2.058, \mathrm{p}=.041$ was shown to be significant, and strengthened interest by academic background $(\mathrm{p}<.05)$ to $\mathrm{F}=.45222, \mathrm{p}=$.Significant as 039 and operational interest by position $(\mathrm{p}<.05)$. It was found to be significant in $\mathrm{p}=0.05$ as $\mathrm{F}=-2.3602$ and $\mathrm{p}=.019$. This means that it is statistically significant based on the significance level of 0.05 .

\section{Conclusions and Suggestions}

The study looked at the interest of teachers participating in gifted education in the national gifted program standards. The results, using the Steps of Interest questionnaire, were shown as awareness, operational, informational, reinforcement, personal, collaborative, and consequential. Although it recognizes the importance of the national gifted program standards, it shows that it is burdensome to apply the national gifted program standards in the actual gifted curriculum to conduct classes and practice them. This means that the direction and principles of the gifted curriculum are understood and agreed, but it is difficult for gifted teachers to practice in the field considering the unfamiliarity and 
understanding of the program standards, and lack of curriculum and activity. In this study, educational support and prescriptions should be provided to meet the interests of gifted teachers in order to revitalize the national gifted program standards. Based on this, the suggestion is as follows.

First, overall, the interest in the national gifted program standard was high, and gifted teachers were positive about the impact and results of the national gifted program standard on students in gifted educational activities. Therefore, it will be necessary to utilize and support specific teacher training and gifted education so that the standards of the national gifted program can be applied to the educational site properly.

Second, a customized national gifted curriculum program should be developed and distributed based on an understanding of the level of interest in the national gifted program standards. Training support and data provision should be prioritized, along with detailed preparation methods, so that teachers in charge of gifted education can write systematic programs rather than composition of fragmented education programs.

Third, for the effective and successful use and settlement of the national gifted program standards, support for the dissemination of materials and training of gifted education programs should be provided to gifted teachers through regular development of gifted teaching.

Such educational efforts will create an atmosphere for active participation in educational sites, a system of educational activities in schools, share educational contents, exchange various teaching activities using new national gifted program standards, and share know-how.

\section{References}

[1] S. D. Lee, The Validity of an Intelligence Scale for the Identification of the Gifted, (2006), Vol.5, No.1, pp.94-113, UCI : G704-001830.2006.5.1.002

[2] Sung-ik Park, Seok-hee Cho, Hong-won Kim, Ji-hyun Lee, Yeo-hong Yoon, Seok-eon Jin, Ki-soon Han, The Theory of Gifted and Talented Education a History of Education and Science, kyoyookbook, (2003)

[3] Ye-won Seo, Min-ki Kim, Ji-eun Park, Hyun-deok Ko, Ah-young Choi, Sang-ga Byun, Ah-reum Kim, Young-seo Lee, Development of the National Gifted Education Program: Elementary and Middle School Science, Korea Educational Development Institute, (2014)

[4] Kyung-ja Kim, Curriculum Innovation:CabAM Based Curriculum Execution Model (CBAM), A History of Education and Science, (1993)

[5] Yong-woon Lee, A Study on the Effectiveness of Support Prescriptions Based on the Level of Interest in the Curriculum of Teachers: Focusing on Cases of Developing Creative Discretionary Activities, Korea University, Doctoral Dissertation, (2004)

[6] Dae-young Moon, Teacher's 'Stages of Concerns' and 'Levels of Use' on STEAM Education, Journal of the Korean Chamber of Education, (2015), Vol.28, No.1, pp.35-52, UCI : G704-000635.2015.28.1.001

[7] Seul-ji Han, Comparison of Curriculum Composition and Analysis and Teacher Recognition of the Department of Gifted Education in Korea, Ewha Womans University Graduate School of Education, Master's thesis, (2012)

[8] Sung-hwan Kim, An Analysis of Gifted Teachers' Interest in the Gifted Curriculum Focuses on the Central Acceptance Model (CBAM), Daegu National University of Education, Master's thesis, (2012)

[9] Sung-hwan Kim, A Study on the Interest of Gifted Teachers in the Gifted Education Process: Focusing on the CBAM (Centric Acceptance Model), Daegu National University of Education, Master's thesis, (2012)

[10] G. E. Hall, S. M. Hord, Implementing Change: Patterns, Principles, and Potholes (2nd Ed), Allyn and Bacon, (2006) 